\title{
Cross-diffusion induced Turing and non-Turing patterns in Rosenzweig-MacArthur model
}

\author{
Nayana Mukherjee, S. Ghorai and Malay Banerjee \\ Department of Mathematics \& Statistics, Indian Institute of Technology, IIT Kanpur, Kanpur, India
}

\begin{abstract}
Pattern formation is widely studied in spatio-temporal preypredator models with only self-diffusion terms. Models with crossdiffusion, besides self-diffusion, take care of the situation in which presence, absence, abundance or scarcity of one species affect the movement of a population of another species in a given domain. Here, we consider cross-diffusion induced pattern formation in a prey-predator model with Rosenzweig-MacArthur type reaction kinetics in a one-dimensional spatial domain. Spatio-temporal prey-predator model with Rosenzweig-MacArthur type reaction kinetics and self-diffusion is unable to generate Turing patterns, rather it produces travelling wave, periodic travelling wave, modulated periodic travelling wave and spatio-temporal chaotic patterns. However, addition of density dependent cross-diffusion leads to satisfaction of Turing instability conditions and generation of stationary Turing patterns. Also, the dynamics of the patterns formed in the selfdiffusion model are preserved. Furthermore, cross-diffusion affects the speed of travelling waves produced in the self-diffusion model. Our focus in this work is to investigate the bifurcation of travelling wave solution into Turing patterns and transition of one pattern into another in the presence of cross-diffusion.
\end{abstract}

\section{ARTICLE HISTORY}

Received 5 October 2018

Accepted 18 February 2019

\section{KEYWORDS}

Cross-diffusion; turing instability; travelling wave; stationary pattern; spatio-temporal chaos; bifurcation

\section{Introduction}

Spatio-temporal pattern formation plays a vital role in understanding the dynamics of interacting populations (such as prey-predator, competing species) as a part of a large ecological community. Such populations are heterogeneously distributed over their habitat which gives rise to the patterns, which may be stationary or non-stationary with respect to time. System of reaction-diffusion equations is used to model such interactions among individuals of more than one species within a domain and the solutions of the system correspond to the patterns. For one spatial dimension, stationary patterns resemble stripes aligned parallel to time axis in a space-time plot (Aragón, Barrio, Woolley, Baker, \& Maini, 2012). When a cross-section of this pattern along the spatial axis is considered at a given time, periodic with respect to space solution is found. The number of peaks formed in such periodic solution increases with an increase in the length of the spatial domain

CONTACT Malay Banerjee malayb@iitk.ac.in $\theta$ Indian Institute of Technology, Kalyanpur, Kanpur, UP 208016, India

(c) 2019 The Author(s). Published by Informa UK Limited, trading as Taylor \& Francis Group

This is an Open Access article distributed under the terms of the Creative Commons Attribution License (http://creativecommons.org/licenses/ by/4.0/), which permits unrestricted use, distribution, and reproduction in any medium, provided the original work is properly cited. 
(Murray, 2001). Non-stationary patterns in one spatial dimension include travelling wave, periodic travelling wave, wave of chaos, etc (Aragón et al., 2012; Dunbar, 1986; Petrovskii \& Malchow, 1999; Sherratt, Eagan, \& Lewis, 1997).

When the homogeneous steady state of a system of reaction-diffusion equations, which is stable under temporal perturbations, becomes unstable due to heterogeneous perturbations, diffusion driven instability or Turing instability sets in Turing (1952). As a result, non-homogeneous in space but stationary with respect to time solutions known as Turing patterns appears. Stationary spot, stripe and mixture of spots and stripes are the few examples of Turing patterns in two spatial dimensions (Cross \& Hohenberg, 1993; Murray, 2001). Non-stationary patterns in two dimensions include spirals, targets, interacting spiral chaos, spatio-temporal chaos, etc.

Travelling waves are transition fronts having constant shape and speed connecting two or more equilibria of the corresponding reaction part of the spatio-temporal model (Sherratt et al., 1997; Shigesada, Kawasaki, \& Teramoto, 1986; Volpert \& Petrovskii, 2009). Existence of travelling waves in prey-predator models is established using various approaches, most common of which are shooting technique, invariant manifold theory and qualitative theory of ordinary differential equations. These were used by Dunbar $(1983,1984,1986)$ and later by others in prey-predator models with various functional responses (Huang, Lu, \& Ruan, 2003; Huang \& Weng, 2013; Li \& Wu, 2008; Lin, Weng, \& Wu, 2011; Peng, Shangbing, \& Yihong, 2017). Schauder's fixed point theory has also been used to prove the existence of travelling waves (Hong \& Weng, 2013; Huang \& Lin, 2014; Zhang, Wang, $\&$ Wang, 2016). Other than these, perturbation technique (Feltham \& Chaplain, 2000), connecting index (Gardner, 1984), comparison principle (Lin, 2014), geometric shooting method (Huang, 2016), etc., are also used to prove the existence of the same. Travelling wave solutions are also found in models for prey taxis (Lee, Hillen, \& Lewis, 2008) and prey-predator systems in environments with unidirectional flow (Hilker \& Lewis, 2010). Existence of travelling waves in a uniform flow supports the choice of one spatial dimension in the corresponding spatio-temporal model.

Spatio-temporal chaos is a fascinating feature of the pattern formation process. In this case, the solutions never settle down to any state and keep on oscillating irregularly with respect to both space and time. It is a more realistic phenomena in the context of ecology. Several numerical techniques have been explored to understand spatio-temporal chaos and to distinguish chaotic behaviour form other solutions. Pascual (1993) used such techniques in a spatio-temporal prey-predator model with Holling type II functional response. A considerable amount of research work has been done on evolution of spatio-temporal chaos from travelling wave or periodic travelling wave in one spatial dimension (Sherratt, 1998; Sherratt et al., 1997; Sherratt, Lambin, \& Sherratt, 2003).

It is obvious that the movement of population of a species in a considered domain cannot be just random. Instead, it is influenced by the presence, absence, abundance, and scarcity of population of another species. To model such a situation, cross-diffusion terms are included into the spatio-temporal population models in addition to self-diffusion terms (Morales et al., 2010; Nathan et al., 2008; Okubo \& Levin, 2013; Potts \& Petrovskii, 2017). Shigesada, Kawasaki, and Teramoto (1979) were the first to work on these terms and they showed that heterogeneity in environment along with non-linear dispersive movements of a population might give rise to pattern formation in systems involving two or more competing species. This approach was later adopted by others 
and the conditions for positivity of solutions for such systems were investigated (Mimura \& Kawasaki, 1980; Oeda, 2011). A competition system with cross-diffusion was approximated by a system of reaction-diffusion equations with linear diffusion terms by Iida, Mimura, and Ninomiya (2006). Gambino, Lombardo, and Sammartino (2013) investigated pattern formation in a cross-diffusion model using weakly nonlinear analysis. Exhaustive numerical simulations were also carried out to explore the effects of cross-diffusion on various interacting population models with self-diffusion (Ling, Zhang, \& Lin, 2014; RuizBaier \& Tian, 2013). These studies have established that inclusion of cross-diffusion makes the modeling approach more realistic.

Several works have been carried out on the existence of travelling waves as well as on emergence of spatio-temporal chaos and other patterns for spatio-temporal prey-predator model with self-diffusion and Holling type II functional response (Dunbar, 1986; Garvie \& Trenchea, 2010; Sherratt et al., 1997). But Turing patterns have not been found for any set of parameter values. Our focus in this work is to introduce a cross-diffusion into the model with self-diffusion and to demonstrate the generation of Turing patterns due to the cross-diffusion term. Furthermore, the effects of cross-diffusion on travelling wave, periodic travelling wave, modulated periodic travelling wave and spatio-temporal chaotic solutions of the self-diffusion model are examined. Our main motive behind this work is to explore the mechanisms for transition of patterns from travelling waves to Turing patterns and chaotic patterns to periodic in time solutions via modulated periodic travelling waves. The temporal model with Holling type II functional response is discussed in Section 2 along with the conditions for Hopf bifurcation. The spatio-temporal model with cross-diffusion is discussed in Section 3 along with the derivation of conditions for Turing instability and existence of travelling wave solutions. Section 4 consists of extensive numerical simulation results and validation of the analytic results of Section 3. Section 5 contains discussion on the results along with some concluding remarks.

\section{The model}

Holling type II functional response was introduced by Holling (1965) and a prey-predator model incorporting this functional response is also known as Rosenzweig-MacArthur model (Rosenzweig \& MacArthur, 1963). It is a modification of the original Lotka-Volterra type prey-predator system where the prey searching and handling time are incorporated into the model. Handling time is the time taken by a predator to catch hold of its prey and prey on it, before it gets ready to search for another prey. The rate of prey capture is saturated when the population of prey is relatively large. Such a model is represented by a system of ordinary differential equations:

$$
\begin{gathered}
\frac{\mathrm{d} N}{\mathrm{~d} t}=r N\left(1-\frac{N}{\kappa}\right)-\frac{\alpha N P}{\alpha h N+1}, \\
\frac{\mathrm{d} P}{\mathrm{~d} t}=\frac{e \alpha N P}{\alpha h N+1}-\mu P,
\end{gathered}
$$

with non-negative initial conditions $N(0) \geq 0, P(0) \geq 0$. Here $r$ and $\kappa$ are the intrinsic growth rate and carrying capacity of the prey population, $\alpha$ is the attack rate of the predator, 
$h$ represents the handling time, $e(0<e<1)$ is the conversion efficiency and $\mu$ represents the death rate of the predators. We take $\bar{N}=N / \kappa, \bar{P}=P / \kappa h r$ and $\bar{t}=r t$ for the dimensionless prey population, predator population and time. Using these in system (1), dropping the over-bars on the dimensionless variables, we obtain

$$
\begin{gathered}
\frac{\mathrm{d} N}{\mathrm{~d} t}=N(1-N)-\frac{N P}{N+c}, \\
\frac{\mathrm{d} P}{\mathrm{~d} t}=\frac{a N P}{N+c}-b P
\end{gathered}
$$

where $a=e / r h, b=\mu$ and $c=1 / \alpha h \kappa$ are all nondimensional positive parameters.

The equilibrium states of system (2) include trivial solution $(0,0)$, axial equilibrium point or the predator free state $(1,0)$ and the coexistence interior equilibrium point $\left(N_{*}=\right.$ $\left.b c /(a-b), P_{*}=\left(1-N_{*}\right)\left(N_{*}+c\right)\right)$ which is feasible for $b(1+c)<a$. From standard linear stability analysis, it can be shown that the equilibrium point $(0,0)$ is always a saddle point. Axial equilibrium point $(1,0)$ is stable and a global attractor for $a /(1+c)<b$ and a saddle point for $a /(1+c)>b$. The interior equilibrium point $\left(N_{*}, P_{*}\right)$ is infeasible in the former case and feasible in the later case. The interior equilibrium point $\left(N_{*}, P_{*}\right)$ loses its stability through a super-critical Hopf-bifurcation giving rise to a stable limit cycle, whose description is given below.

\subsection{Hopf bifurcation}

The conditions for local asymptotic stability and Hopf bifurcation of the coexistence steady state of system (2) are discussed here. For the linear stability analysis, we write

$$
N_{1}=N-N_{*}, \quad P_{1}=P-P_{*}, \quad\left|N_{1}\right| \ll 1, \quad\left|P_{1}\right| \ll 1,
$$

which on substituting into (2) and linearizing gives

$$
\left(\begin{array}{l}
N_{1 t} \\
P_{1 t}
\end{array}\right)=\left(\begin{array}{cc}
-N_{*}+\frac{N_{*} P_{*}}{\left(N_{*}+c\right)^{2}} & -\frac{N_{*}}{\left(N_{*}+c\right)} \\
\frac{a c P_{*}}{\left(N_{*}+c\right)^{2}} & 0
\end{array}\right)\left(\begin{array}{l}
N_{1} \\
P_{1}
\end{array}\right) \equiv\left(\begin{array}{cc}
a_{11} & a_{12} \\
a_{21} & 0
\end{array}\right)\left(\begin{array}{l}
N_{1} \\
P_{1}
\end{array}\right) .
$$

The conditions for asymptotic stability of $\left(N_{*}, P_{*}\right)$ are $a_{11}<0$ and $a_{12} a_{21}<0$ which are automatically satisfied (Murray, 2001). Hopf bifurcation occurs when $\left(N_{*}, P_{*}\right)$ becomes unstable to temporal perturbations giving periodic solutions. At the Hopf threshold, the linearized system's eigenvalues are a complex conjugate pair with zero real parts. Taking $b$ to be the bifurcation parameter for the model (2), we write the complex conjugate eigenvalues of the Jacobian matrix as $\lambda(b), \bar{\lambda}(b)=\lambda_{1}(b) \pm i \lambda_{2}(b)$. Thus, Hopf bifurcation occurs at $b=b_{H}$ if

$$
\lambda_{1}\left(b_{H}\right)=0, \quad \lambda_{2}\left(b_{H}\right)>0 \quad \text { and } \quad \frac{\partial}{\partial b} \lambda_{1}\left(b_{H}\right) \neq 0 .
$$

The Hopf bifurcation threshold $b_{H}$ for model (2) can be obtained by solving

$$
a_{11}(b)=0,
$$


which is explicitly obtained as

$$
b_{H}=\frac{a(1-c)}{(1+c)} .
$$

For values of $b<b_{H}$, the interior equilibrium is unstable and stable for $b_{H}<b<$ $a /(1+c)$ and $c<1$. From the first Liapunov number (Perko, 2013), which is negative in this case, we can say that the limit cycle, generated due to Hopf instability, is stable. Hence, the Hopf bifurcation is supercritical.

\section{The spatio-temporal model}

Now we include the spatial aspect into the temporal model (2) by extending it to a coupled reaction-diffusion equations over a bounded one dimensional spatial domain. A similar modeling approach was followed in Petrovskii and Malchow (1999), where the authors were interested to find a minimal spatio-temporal model showing spatial pattern and its dependence on various initial conditions. Let $N(x, t)$ and $P(x, t)$ respectively denote the dimensionless prey and predator population densities at position $x \in[0, L]$ and time $t \geq 0$. Self-diffusion terms are used to model the random movement of the prey and predator individuals. The corresponding spatio-temporal extension of the prey-predator model with Holling type II functional response is given by

$$
\begin{gathered}
\frac{\partial N}{\partial t}=N(1-N)-\frac{N P}{N+c}+\frac{\partial^{2} N}{\partial x^{2}}, \\
\frac{\partial P}{\partial t}=\frac{a N P}{N+c}-b P+d_{1} \frac{\partial^{2} P}{\partial x^{2}},
\end{gathered}
$$

subject to the non-negative initial conditions and no-flux boundary conditions

$$
\begin{aligned}
& N(x, 0) \geq 0, P(x, 0) \geq 0, \quad x \in(0, L), \\
& \frac{\partial N}{\partial x}=\frac{\partial P}{\partial x}=0, \quad x=0, x=L, t \geq 0 .
\end{aligned}
$$

Here $d_{1}$ is a dimensionless quantity representing the ratio of diffusivities of predator over prey. Due to nondimensionalization, the rate of self-diffusion for prey population is 1. Model (6) cannot produce Turing patterns, however it can produce travelling wave, periodic travelling wave and spatio-temporal chaos under proper parameteric restrictions (Petrovskii \& Malchow, 1999; Sherratt et al., 1997). Now, we incorporate the effects of presence, abundance or scarcity of the prey population over the predator population to explore if the modified model can produce other type of patterns, mainly Turing patterns. Let the movement of the predator population depends on the prey population gradient. To model this situation, we incorporate density dependent cross-diffusion for predator into the selfdiffusion model (6). It describes the tendency of the predator species to keep safe distance from high density areas of the prey, preferring low-density areas of preys for hunting (Gambino, Lombardo, \& Sammartino, 2018). Such modeling has also been done in Ruiz-Baier 
and Tian (2013), Liu and Lin (2010), Peng and Zhang (2016). Hence, the modified model with cross-diffusion terms is of the form

$$
\begin{gathered}
\frac{\partial N}{\partial t}=N(1-N)-\frac{N P}{N+c}+\frac{\partial^{2} N}{\partial x^{2}}, \\
\frac{\partial P}{\partial t}=\frac{a N P}{N+c}-b P+d_{1} \frac{\partial^{2} P}{\partial x^{2}}+d_{2} \frac{\partial^{2}(N P)}{\partial x^{2}},
\end{gathered}
$$

where $d_{2}$ is the dimensionless cross-diffusion coefficient. The initial and boundary conditions are same as given in (7). There are other modeling approaches too as described in Okubo and Levin (2013). Now we shall derive the conditions for the existence of travelling waves solutions and Turing instability for the model (8) in two consecutive subsections.

\subsection{Existence of travelling wave solution}

A travelling wave is a wave which travels without changing its shape joining two or more equilibrium points of the reaction part of the spatio-temporal model (Murray, 2001). Note that $N(x, t) \equiv N_{*}>0, P(x, t) \equiv P_{*}>0$ are taken to be the homogeneous steady state for (8) since they satisfy system (8) along with the initial and boundary conditions. Therefore, if a solution $u(x, t)$ represents a travelling wave, the shape of the solution will be same with advancement of time and the speed of propagation, denoted by $s$, will be same at all time. To find such a solution for the model with cross-diffusion $(8)$, we substitute $N(x, t) \equiv$ $U(x-s t)$ and $P(x, t) \equiv V(x-s t)$ which gives

$$
\begin{gathered}
-s U^{\prime}=F(U, V)+U^{\prime \prime}, \\
-s V^{\prime}=G(U, V)+d_{1} V^{\prime \prime}+d_{2}\left(U V^{\prime \prime}+U^{\prime \prime} V+2 U^{\prime} V^{\prime}\right),
\end{gathered}
$$

where

$$
\begin{aligned}
& F(U, V)=U(1-U)-\frac{U V}{U+c}, \\
& G(U, V)=\frac{a U V}{U+c}-b V .
\end{aligned}
$$

Taking $U^{\prime}=W$ and $V^{\prime}=X$, we convert the system of ordinary differential Equations (9) into a system of first order ordinary differential equations

$$
\begin{gathered}
U^{\prime}=W, \\
V^{\prime}=X, \\
W^{\prime}=-s W-F(U, V), \\
X^{\prime}=\frac{1}{d_{1}+d_{2} U}\left(-s X-G(U, V)-2 d_{2} W X+s d_{2} V W+d_{2} V F(U, V)\right) .
\end{gathered}
$$

From the linear stability analysis of the system of ordinary differential Equation (10) around the equilibrium points, we find out the criteria for existence of travelling wave 
connecting the homogeneous steady-states of the system (8). Also, the effects of crossdiffusion on the speed of the travelling wave can be studied. The equilibrium points of the system of ordinary differential Equation (10) are $E_{0}(0,0,0,0), E_{1}(1,0,0,0)$ and $E_{2}\left(b c /(a-b), a c(-b c+a-b) /(a-b)^{2}, 0,0\right)$. From the characteristic equation of the Jacobian matrix for each of these equilibrium points, we calculate the eigenvalues based on which we derive the conditions for existence of heteroclinic connections between two equilibrium points. The Jacobian matrix of the system $(10)$ at $E_{0}(0,0,0,0)$ is

$$
\mathbf{J}_{E_{0}}=\left(\begin{array}{cccc}
0 & 0 & 1 & 0 \\
0 & 0 & 0 & 1 \\
-1 & 0 & -s & 0 \\
0 & \frac{b}{d_{1}} & 0 & -\frac{s}{d_{1}}
\end{array}\right)
$$

which have eigenvalues $-s / 2 \pm \sqrt{s^{2}-4} / 2$ and $\left(-s \pm \sqrt{4 b d_{1}+s^{2}}\right) / 2 d_{1}$. It is clear from the eigenvalues that one-dimensional unstable manifold and three-dimensional stable manifold exist at $E_{0}$ for $s \geq 2$. It should be mentioned here that we do not consider the case of $s<2$ since it leads to complex eigenvalues. In this case, the trajectories will spiral in or out of $E_{0}$, which gives rise to negative values for the population density. In order to maintain the feasibility of the situation that is non-negativity of population densities of both prey and predator, we consider the cases where $s \geq 2$. Cross-diffusion parameter $d_{2}$ has no effect on the stability of $E_{0}$ since the eigenvalues of $\mathbf{J}_{E_{0}}$ are independent of $d_{2}$.

At $E_{1}(1,0,0,0)$ which may connect to $E_{2}$, the Jacobian matrix is of the form,

$$
\mathbf{J}_{E_{1}}=\left(\begin{array}{cccc}
0 & 0 & 1 & 0 \\
0 & 0 & 0 & 1 \\
1 & \frac{1}{1+c} & -s & 0 \\
0 & \frac{b c-a+b}{\left(d_{1}+d_{2}\right)(1+c)} & 0 & -\frac{s}{\left(d_{1}+d_{2}\right)}
\end{array}\right) .
$$

The eigenvalues of $\mathbf{J}_{E_{1}}$ are $-s / 2 \pm \sqrt{s^{2}-4} / 2$ and

$$
\frac{1}{(c+1)\left(d_{1}+d_{2}\right)}\left(-s(1+c) \pm \sqrt{s^{2}(1+c)^{2}-\left(d_{1}+d_{2}\right)(1+c)(4 a-4 b(1+c))}\right) .
$$

From the condition $b(1+c)<a$ for feasibility of $\left(N_{*}, P_{*}\right)$, we have $s^{2} \geq 4\left(d_{1}+d_{2}\right)$ $(a-b(1+c)) /(1+c)$ which clearly shows that the minimum speed of propagation $s_{\min }$ increases for $d_{2}>0$. From the eigenvalues, it is clear that four-dimensional stable manifold exist at $E_{1}$ for $s \geq 2$ and $b(1+c)<a$.

The explicit forms of the eigenvalues of the Jacobian matrix $\mathbf{J}_{E_{2}}$ are algebraically complicated which are not presented here. These will be discussed with a suitable numerical example in Section 4.2. The conditions for existence of the travelling waves derived here will be numerically validated in that section too with the help of simulations.

\subsection{Turing instability}

Turing instability occurs when the homogeneous steady-state is stable to infinitesimal perturbations in the absence of diffusion but becomes unstable due to infinitesimal spatial 
perturbations in the presence of diffusion (Murray, 2001; Turing, 1952). The conditions for stability to perturbations in absence of diffusion are $a_{11}<0$ and $a_{12} a_{21}<0$ discussed before. We perturb the homogeneous steady state $\left(N_{*}, P_{*}\right)$ of system $(8)$ as

$$
\left(\begin{array}{l}
N \\
P
\end{array}\right)=\left(\begin{array}{l}
N_{*} \\
P_{*}
\end{array}\right)+\left(\begin{array}{l}
C_{1} \\
C_{2}
\end{array}\right) e^{\lambda t} \cos k x,
$$

where $0<C_{1}, C_{2} \ll 1, \lambda$ is the growth rate of perturbations and $k$ is the wave number. Substituting (13) into (8), the characteristic equation for the growth rate $\lambda$ is found from $\operatorname{Det}\left(\mathbf{J}_{1}\right)=0$, where

$$
\mathbf{J}_{1}=\left(\begin{array}{cc}
a_{11}-k^{2}-\lambda & a_{12} \\
a_{21}-d_{2} P_{*} k^{2} & -\left(d_{1}+d_{2} N_{*}\right) k^{2}-\lambda
\end{array}\right) .
$$

The explicit expressions for $a_{11}, a_{12}$ and $a_{21}$ are given in (3). The characteristic equation is given by

$$
\lambda^{2}-\lambda\left(a_{11}-k^{2}\left(1+d_{1}+d_{2} N_{*}\right)\right)+h\left(k^{2}\right)=0,
$$

where

$$
h\left(k^{2}\right)=\left(d_{1}+d_{2} N_{*}\right) k^{4}+\left(d_{2} P_{*} a_{12}-d_{2} N_{*} a_{11}-d_{1} a_{11}\right) k^{2}-a_{12} a_{21} .
$$

Equating the derivative $d h\left(k^{2}\right) / d\left(k^{2}\right)$ of $h\left(k^{2}\right)$ with respect to $k^{2}$ to zero at $k=k_{T}$, which is the critical wave number for Turing instability, gives

$$
k_{T}^{2}=\frac{d_{1} a_{11}+d_{2} N_{*} a_{11}-d_{2} P_{*} a_{12}}{2\left(d_{1}+d_{2} N_{*}\right)} .
$$

It should be noted that for $d_{2}=0$, i.e. for the self-diffusion model (6), $a_{11}$ has to be positive to maintain the positivity of $k_{T}^{2}$. But from the conditions for Turing instability, we know that $a_{11}<0$ is required for the stability of homogeneous steady state under temporal perturbation. Thus, Turing instability conditions can not be satisfied for the self-diffusion model (6).

We find the Turing bifurcation condition for the cross-diffusion model (8) by substituting the expression for $k_{T}^{2}$ in $h\left(k^{2}\right)=0$. From this, we obtain the Turing bifurcation threshold $d_{2}$ explicitly as

$$
\begin{aligned}
d_{2}^{T}= & \frac{1}{N_{*}^{2} a_{11}^{2}-2 N_{*} P_{*} a_{11} a_{12}+P_{*}^{2} a_{12}^{2}}\left[d_{1} P_{*} a_{11} a_{12}-d_{1} N_{*} a_{11}^{2}-2 N_{*} a_{12} a_{21}\right. \\
& \left.+2 \sqrt{d_{1} N_{*} P_{*} a_{11} a_{12}^{2} a_{21}-d_{1} P_{*}^{2} a_{12}^{3} a_{21}+N_{*}^{2} a_{12}^{2} a_{21}^{2}}\right]
\end{aligned}
$$

whenever the expression is feasible. The wave number $k_{T}$ of the most rapidly growing eigen mode on account of the boundary conditions satisfies $p \pi / L \leq k_{T} \leq q \pi / L$, where $p$ and $q$ are natural numbers and $L$ is the length of the domain. Therefore, the expected wavelength is of the form $\chi=2 \pi / m$, where $m$ is a natural number satisfying $p \leq m \leq q$. The total number of stripes in the spatial domain will be $L / \chi$ (Aragón et al., 2012). These results can be numerically verified with suitable examples which are described in Section 4.3. 


\section{Simulation results}

We have obtained the temporal Hopf bifurcation threshold in terms of $b$ and Turing bifurcation threshold in terms of $d_{2}$ for system (8) in the previous section. We now perform numerical simulations to understand the pattern formation scenario of system (8). Before that, we discuss the same for the self-diffusion model (6). For this, we consider a onedimensional spatial domain $[0,1200]$ with $\Delta x=1$ and $\Delta t=0.01$. Results are verified with other choices of $\Delta x$ and $\Delta t$ and we can assure that the reported results are free from numerical artefacts. Three-point stencil finite difference scheme for the diffusion part and forward Euler method for the temporal part are used to simulate the models (6) and (8). Values of parameters $c=0.2$ and $d_{1}=2$ are kept fixed for all the simulations. Along with no-flux boundary conditions, initial conditions for the simulations are taken to be

$$
N(x, 0)=N_{*}, \quad P(x, 0)= \begin{cases}P_{*} & \text { if }|\mathrm{x}-600|<5, \\ 0 & \text { otherwise. }\end{cases}
$$

Now we discuss the patterns exhibited by model (6) followed by those produced by simulating model (8) in the following subsections. We also validate the analytical results with the numerical examples and examine the effects of cross-diffusion on pattern formation scenario of the self-diffusion model (6).

\subsection{Patterns without cross-diffusion}

The conditions for Turing instability are not satisfied for prey-predator model with Holling type II functional response in presence of self-diffusion and numerical simulations with any set of parameter values do not produce Turing patterns. But there are other patterns exhibited by with self-diffusion model (6).

For the set of parameters $a=0.6, b=0.35$ close to the Hopf bifurcation threshold $b_{H}=$ 0.4 , the transient state consists of periodic travelling wave which are formed till the solution starting from the given initial condition reaches the boundary (see Figure 1(a,b)). After a long time, it becomes a periodic in space and time solution which finally leads to periodic in time solution (see Figure 1(c,d)). Similar results are found for the parameters near the Hopf bifurcation boundary.

For parameters $a=0.9, b=0.2$, that are away from the Hopf bifurcation threshold $b_{H}=0.6$, modulated periodic travelling wave followed by chaotic pattern appears. The symmetry in the initial conditions is maintained for a long time and then symmetry breaks down giving rise to chaotic oscillations (see Figure 2). Similar results are found for other parameter values, away from the temporal Hopf bifurcation boundary.

We now consolidate all such simulation results in a bifurcation diagram plotted on $a-b$ plane (see Figure 3). The dashed curve divides the domain into two parts, the upper part corresponds to region I where the coexistence equilibrium point $\left(N_{*}, P_{*}\right)$ is not feasible. $\left(N_{*}, P_{*}\right)$ is feasible in the lower part of the domain which is divided by the black curve $b_{H}=0.6667 a$ corresponding to Hopf bifurcation boundary. Region II lies between the red and black curves where the homogeneous steady state $\left(N_{*}, P_{*}\right)$ is stable under temporal perturbations. Travelling waves are formed in region II connecting the total extinction state $(0,0)$, predator free state $(1,0)$ and co-existing steady state $\left(N_{*}, P_{*}\right)$ with the given initial conditions. Region III lies just below the Hopf bifurcation curve where periodic travelling 


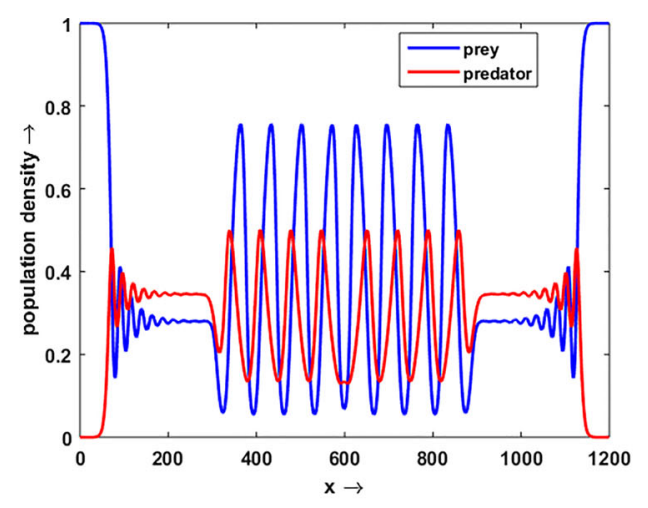

(a)

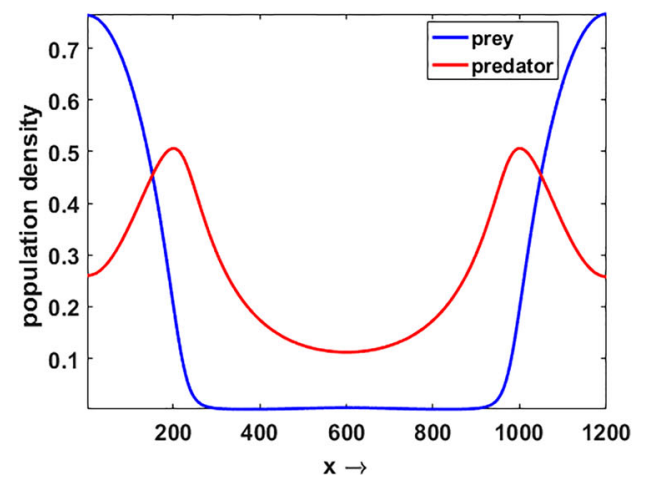

(c)

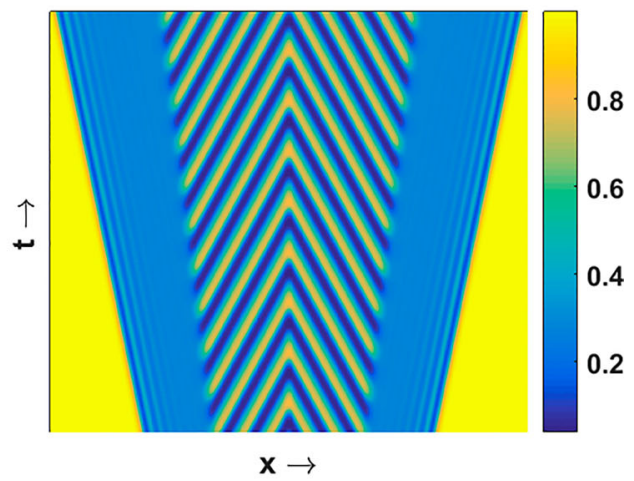

(b)

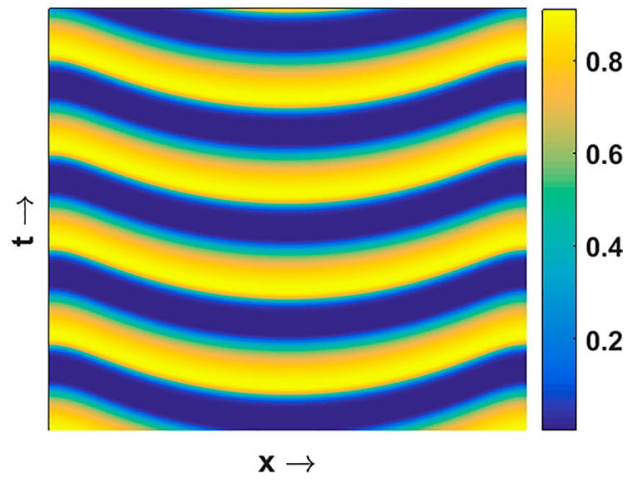

(d)

Figure 1. Periodic travelling wave followed by periodic with respect to space and time solutions for parameter values $a=0.6, b=0.35, c=0.2, d_{1}=2, d_{2}=0$ : (a) Plot of $u(x, t)$ and $v(x, t)$ at time $t=1500$; (b) Plot of patterns formed in $x$-t plane till time $t=1500$; (c) Plot of $u(x, t)$ and $v(x, t)$ at time $t=4500$;

(d) Plot of patterns formed in $x$-t plane till time $t=4500$.

waves emerge initially followed by periodic in space and time solution and finally periodic in time solutions (see Figure 1). Region IV lies below region III away from the Hopf bifurcation boundary, where modulated periodic travelling waves appear initially which ultimately turn into chaotic patterns.

We shall now introduce cross-diffusion and examine the changes occurring to each of the solutions generated by corresponding parameter sets of the $a-b$ plane in Figure 3 . We will also compare the patterns generated in the self-diffusion model (6) with those of the cross-diffusion model (8).

\subsection{Travelling waves due to cross-diffusion}

Here, we discuss the effects of cross-diffusion on travelling wave solutions of the selfdiffusion model (6) with the help of a numerical example. We consider the parameter set $a=0.9, b=0.65, c=0.2, d_{1}=2$. We take $d_{2}=0$ first and then gradually increase it to note the changes due to the influence of cross-diffusion. We have already converted 


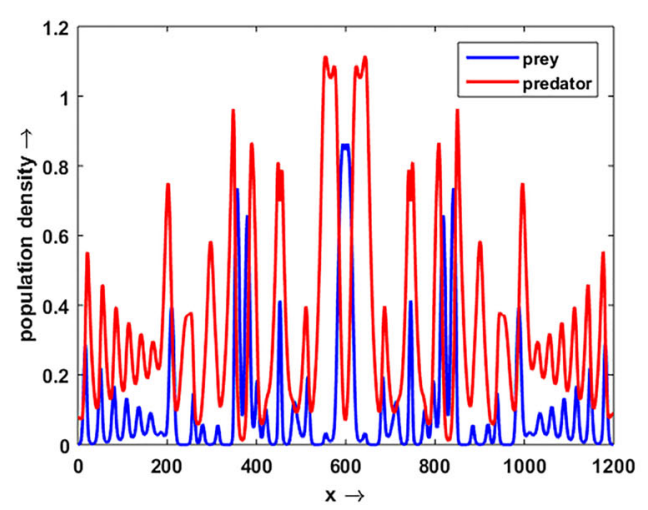

(a)

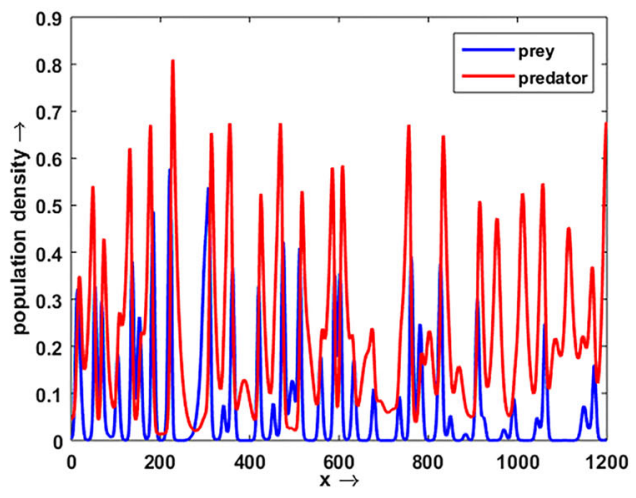

(c)

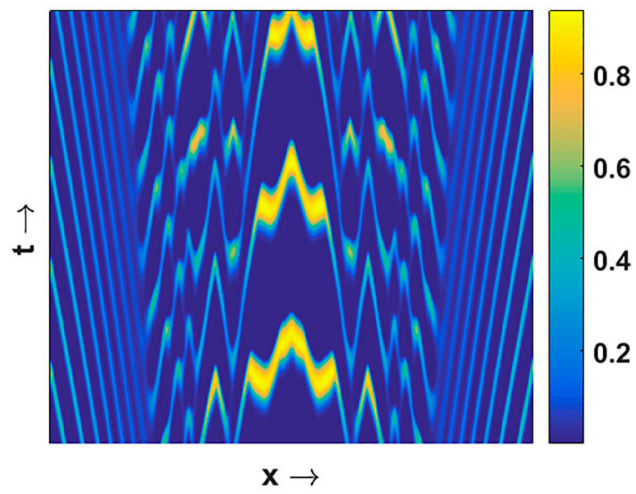

(b)

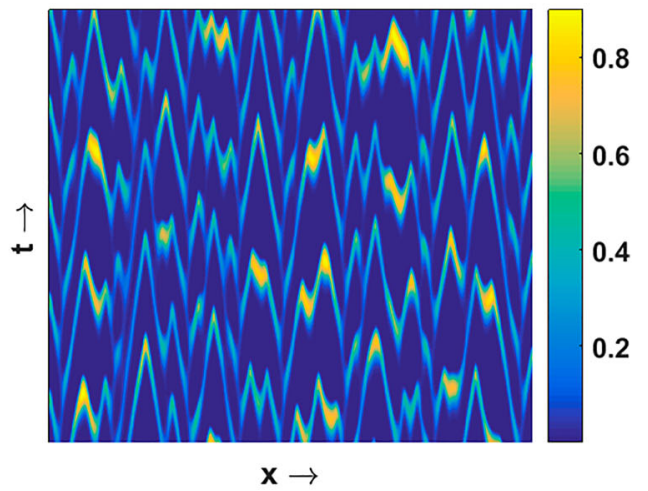

(d)

Figure 2. Modulated periodic travelling wave followed by chaotic pattern for parameter values $a=0.9$, $b=0.2, c=0.2, d_{1}=2, d_{2}=0$ : (a) Plot of $u(x, t)$ and $v(x, t)$ at time $t=1500$; (b) Plot of patterns formed in $x$-t plane till time $t=1500$; (c) Plot of $u(x, t)$ and $v(x, t)$ at time $t=4500$; (d) Plot of patterns formed in $x$ - $t$ plane till time $t=4500$.

the model with cross-diffusion (8) to a system of ordinary differential Equation (10) by substitution of $N(x, t) \equiv U(x-s t)$ and $P(x, t) \equiv V(x-s t)$.

From the linear stability analysis of system (10) around its equilibrium points, we find the criteria for existence of travelling wave connecting the equilibrium points of the temporal part of the model. Also the effects of cross-diffusion on the speed of the travelling wave can be studied. The three equilibrium points of the form $(U, V, W, X)$ of system (10) with the given parameter values are $E_{0} \equiv(0,0,0,0), E_{1} \equiv(1,0,0,0), E_{2} \equiv(0.52,0.3456,0,0)$.

The eigenvalues of Jacobian matrix $\mathbf{J}_{E_{0}}$ of system $(10)$ at $E_{0}(0,0,0,0)$ are $\lambda_{1,2}=-0.5 s \pm$ $0.5 \sqrt{s^{2}-4}$ and $\lambda_{3,4}=-0.25 s \pm 0.05 \sqrt{25 s^{2}+130}$. Thus, it is clear that there exists a onedimensional unstable manifold at $E_{0}$ for $s \geq 2$, which creates a heteroclinic connection with one of the other equilibrium points $E_{1}$ or $E_{2}$. The characteristic polynomial of the Jacobian matrix $\mathbf{J}_{E_{1}}$ of system $(10)$ at $E_{1}(1,0,0,0)$ is

$$
\begin{aligned}
P_{E_{1}}(\lambda)= & \left(1.2 d_{2}+2.4\right) \lambda^{4}+\left(3.6 s+1.2 s d_{2}\right) \lambda^{3}+\left(1.2 s^{2}-1.2 d_{2}-2.28\right) \lambda^{2} \\
& -1.08 s \lambda-0.12
\end{aligned}
$$




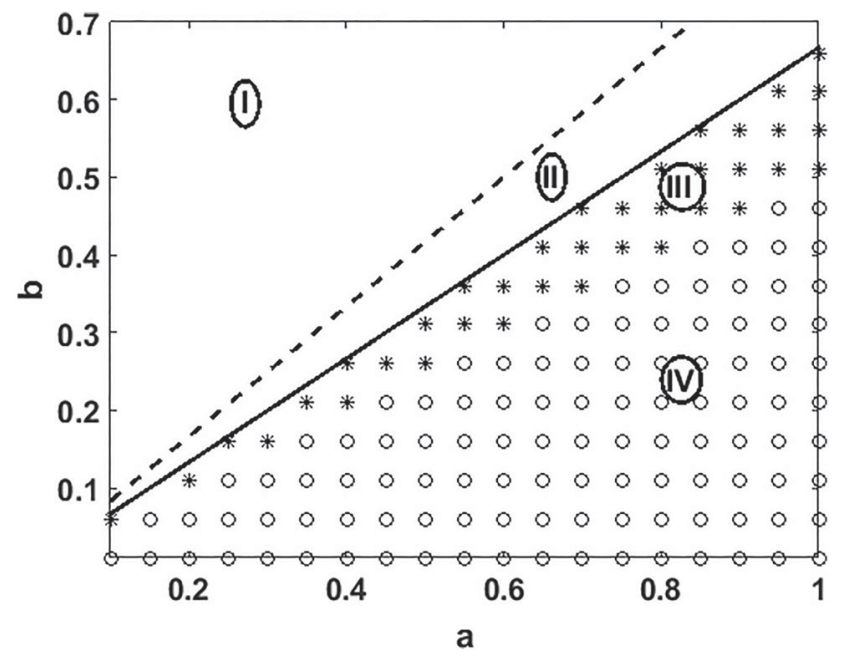

Figure 3. Bifurcation curves in $a-b$ plane: dashed curve marks the boundary of region I where the coexistence equilibrium point is not feasible; black curve represents the Hopf bifurcation curve; region II lies between the red and black curve where coexistence equilibrium point $\left(N_{*}, P_{*}\right)$ is feasible and temporally stable; ${ }^{* * \prime}$ symbols represent region III where periodic travelling waves are formed followed by periodic in time solutions; ' $o$ ' symbols represent region IV where chaotic patterns are observed.

The eigenvalues are $\lambda_{1,2}=-0.5 s \pm 0.5 \sqrt{s^{2}+4}$ and $\lambda_{3,4}=\left(0.1 /\left(d_{2}+2\right)\right)(-5 s \pm$ $\left.\sqrt{25 s^{2}-10 d_{2}-20}\right)$. It is obvious that $\lambda_{1}$ is always positive while $\lambda_{2}$ is always negative. If $s \geq \sqrt{10 d_{2}+20} / 5=s_{\min }$, then $\lambda_{3}$ and $\lambda_{4}$ are real negative eigenvalues. This becomes clear form the plot of $P_{E_{1}}$, which shows that for $s \geq s_{\min }$, three of the roots are real and negative while the fourth one is real and positive (see Figure $4(\mathrm{a}, \mathrm{b})$ ). Hence, there will be a onedimensional unstable manifold and a three-dimensional stable manifold at equilibrium $E_{1}$. If $0<s<\sqrt{10 d_{2}+20} / 5$, then $\lambda_{3}$ and $\lambda_{4}$ are a pair of complex conjugate eigenvalues with negative real part. $E_{1}$ then becomes a spiral point which is biologically not feasible. Therefore, if a travelling wave solution of system (10) exists, then the possible minimum

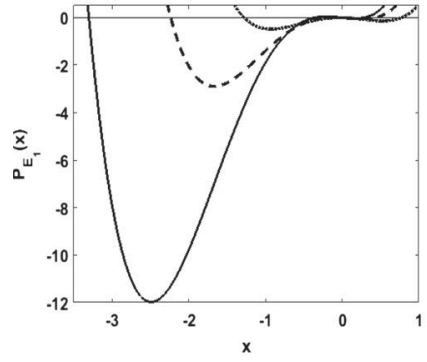

(a)

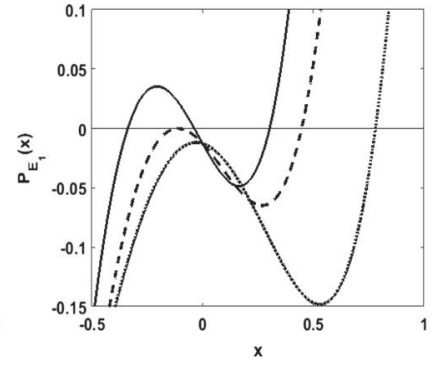

(b)

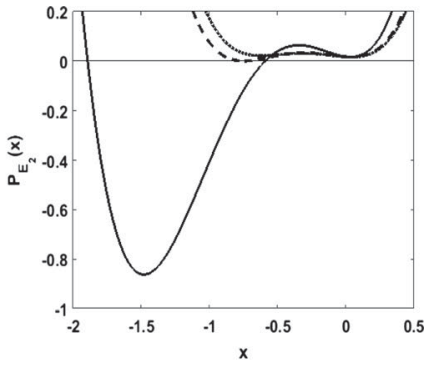

(c)

Figure 4. (a) Plot of the characteristic polynomial $P_{E_{1}}$ for the parameter values $a=0.9, b=0.65, c=$ $0.2, d_{1}=2, d_{2}=0$; (b) zoomed version of (a); (c) Plot of the characteristic polynomial $P_{E_{2}}$. Solid, dashed and dotted curves represent $s>s_{\min }, s=s_{\min }$ and $s<s_{\min }$ respectively. 
speed for biologically relevant solution with non-negative $U$ and $V$ is

$$
s_{\min }=\frac{\sqrt{10 d_{2}+20}}{5} .
$$

It is clear that the minimum speed $s_{\min }$ of the travelling wave increases with an increase in value of the cross-diffusion parameter $d_{2}$. Travelling wave solutions for two different values of $d_{2}$ are shown in Figure 5. The travelling wave of Figure 5(b) with non-zero cross-diffusion $d_{2}=4$ moves faster than the one in Figure 5(a) with zero cross-diffusion $d_{2}=0$.

At the non-trivial equilibrium $E_{2} \equiv(0.52,0.3456,0,0)$, the Jacobian matrix is of the form

$$
\begin{aligned}
& \mathbf{J}_{E_{2}} \\
& =\left(\begin{array}{cccc}
0 & 0 & 1 & 0 \\
0 & 0 & 0 & 1 \\
0.1733 & 0.7222 & -s & 0 \\
\frac{-0.0864+0.043 d_{2}}{0.3744 d_{2}+1.44} & -\frac{0.1797 d_{2}}{0.3744 d_{2}+1.44} & \frac{0.2488 s d_{2}}{0.3744 d_{2}+1.44} & -\frac{0.72 s}{0.3744 d_{2}+1.44}
\end{array}\right) .
\end{aligned}
$$

For $s \geq \sqrt{10 d_{2}+20} / 5$, the plot of the corresponding characteristic polynomial $P_{E_{2}}$ in Figure 4 (c) shows that the $P_{E_{2}}$ has exactly two negative real roots. By calculation the other two roots are found to be complex with positive real parts. Thus, there is a two-dimensional unstable manifold at the equilibrium $E_{2}$. As a result, a heteroclinic connection is possible between $E_{0}, E_{1}$ and $E_{2}$ of system (10). Figure 5 depicts the travelling wave connecting the equilibrium points $(1,0)$ and $\left(N_{*}, P_{*}\right)$ of the spatio-temporal model $(8)$. If we change the initial conditions to

$$
N(x, 0)=\left\{\begin{array}{ll}
N_{*} & \text { if }|\mathrm{x}-600|<5 \\
0 & \text { otherwise }
\end{array}, \quad P(x, 0)= \begin{cases}P_{*} & \text { if }|\mathrm{x}-600|<5 \\
0 & \text { otherwise }\end{cases}\right.
$$

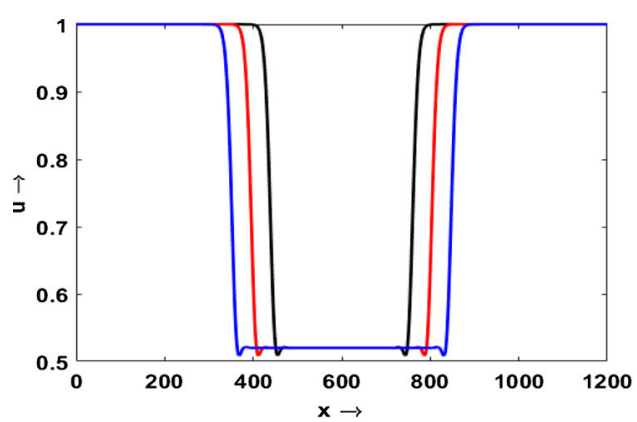

(a)

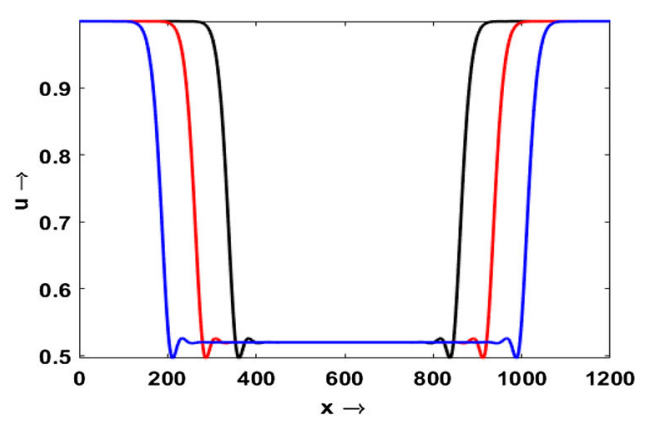

(b)

Figure 5. Travelling wave profiles connecting two equlibrium points: (a) $a=0.9, b=0.65, c=$ $0.2, d_{1}=2, d_{2}=0$ at time $t=100$ (black), 200 (red), and 300 (blue); (b) $a=0.9, b=0.65, c=0.2, d_{1}=$ $2, d_{2}=4$, at time $t=100$ (black), 200 (red), and 300 (blue). 


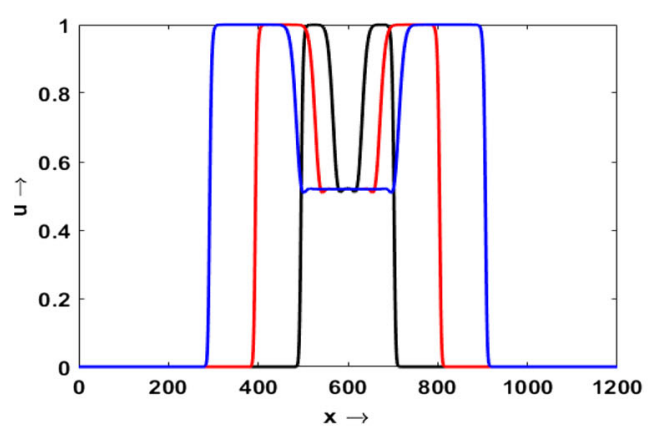

(a)

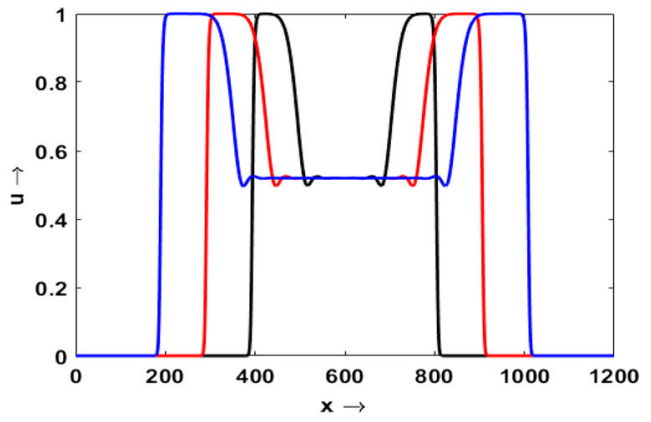

(b)

Figure 6. Travelling wave profiles connecting three equilibrium points for (a) $a=0.9, b=0.65, c=$ $0.2, d_{1}=2, d_{2}=0$ at time $t=100$ (black), 200 (red), and 300 (blue); (b) $a=0.9, b=0.65, c=0.2, d_{1}=$ $2, d_{2}=4$, at time $t=100$ (black), 200 (red), and 300 (blue).

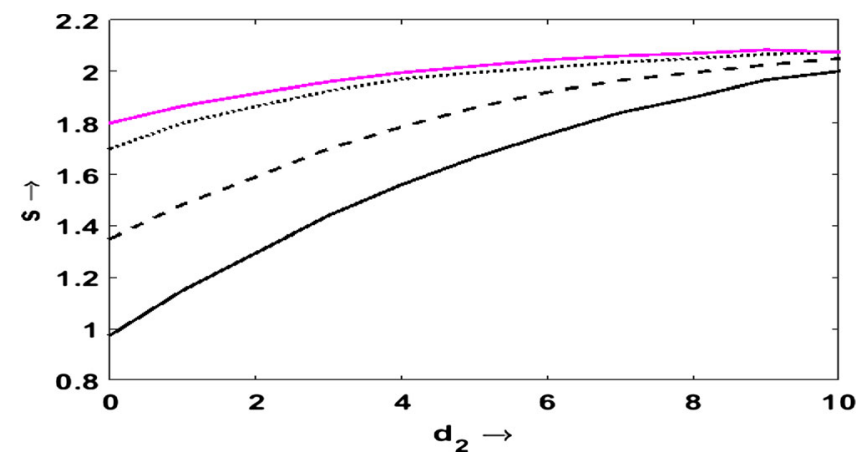

Figure 7. Speed of travelling wave $s$ with respect to cross-diffusion parameter $d_{2}$ for $d_{1}=2$ (black curve), $d_{1}=4$ (black dashed curve), $d_{1}=6$ (black dotted curve), $d_{1}=8$ (magenta curve).

then we get travelling waves connecting all the three homogeneous steady-states $(0,0)$, $(1,0)$ and $\left(N_{*}, P_{*}\right)$ of model $(8)$ as shown in Figure 6 . In this case also, the speed of the travelling wave increases with increase in the cross-diffusion parameter $d_{2}$ when the plots are obtained at same instances of time.

Thus, heteroclinic connections between the the total extinction state, predator free state and co-existing homogeneous steady state exist in both the systems with self- and crossdiffusion. The travelling wave of system with cross-diffusion (8) moves faster than that of the self-diffusion model (6). The dependence of the speed of travelling wave on the cross-diffusion coefficient $d_{2}$ for a given value of self-diffusion coefficient $d_{1}$ is shown in Figure 7. For a given $d_{1}$, the speed of the travelling wave increases as $d_{2}$ is increased from 0 but ultimately it reaches a saturation level. Furthermore, the speed of the travelling wave is almost independent of cross-diffusion coefficient $d_{2}$ for higher values of $d_{1}$.

\subsection{Cross-diffusion induced turing patterns}

In the presence of cross-diffusion, Turing patterns are produced in system (8). We discuss this phenomenon with a numerical example. Considering parameter values $a=0.4$, 


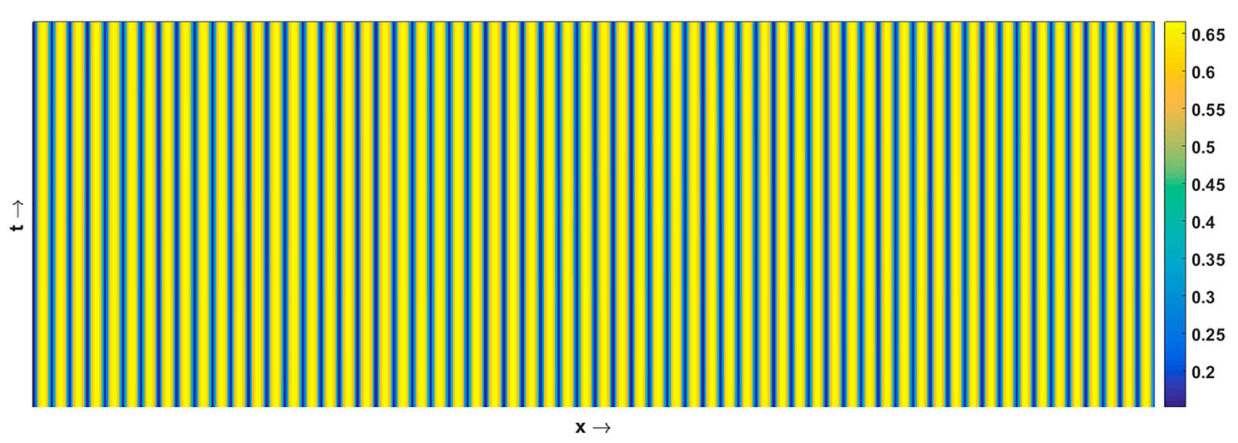

(a)

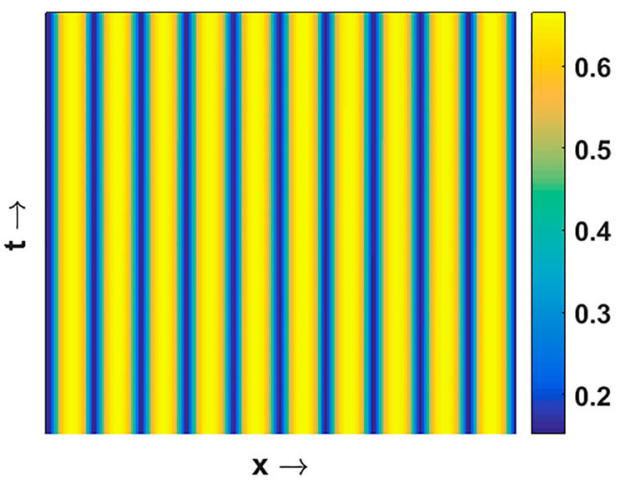

(b)

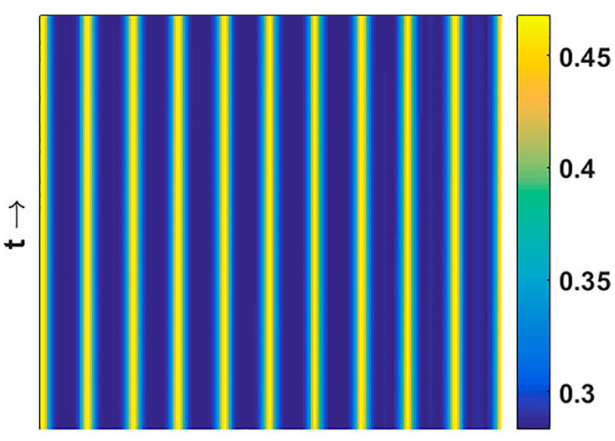

$x \rightarrow$

(c)

Figure 8. Turing pattern for parameter values $a=0.4, b=0.27, c=0.2, d_{1}=2, d_{2}=6$ : (a) plot of $u(x, t)$ till time $t=1500$; (b) zoomed version of (a) for $L=200$; (c) Turing patterns for $L=200$ for the predator population.

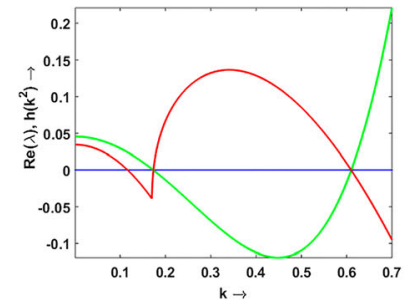

(a)

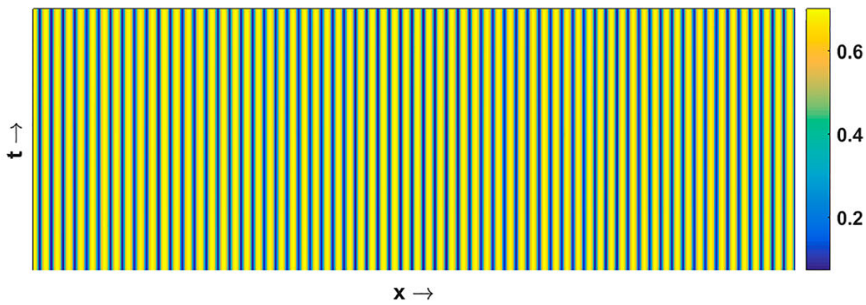

(b)

Figure 9. (a) Plot of $\operatorname{Re}(\lambda)$ (red curve) and $h\left(k^{2}\right)$ (green curve) with respect to $k$; (b) plot of Turing pattern for prey population in $x$ - $t$ plane till time $t=1500$. Parameter values are $a=0.3, b=0.19, c=0.2, d_{1}=2$ and $d_{2}=6$.

$b=0.27, c=0.2$ and $d_{1}=2$ for which the Turing bifurcation threshold from (17) is $d_{2}^{T}=3.8463$ and $k_{T}=0.34558$. Turing patterns are formed for $d_{2}>d_{2}^{T}$ and Figure 8 depicts the same for $d_{2}=6$. For validating the analytic results with the numerical simulation results, the corresponding eigen mode that grows rapidly has wavelength $2 \pi / k_{T}=$ 19.67 and the approximate number of stripes to be found due to Turing instability is $\frac{1200}{19.67} \approx 61$. We observe 61 stripes for simulation with the given initial conditions (18) (see 
Figure 8(a)). Turing patterns with 61 stripes are also found for random perturbation around the homogeneous steady state $\left(N_{*}, P_{*}\right) \equiv(0.4153,0.3597)$. Zoomed version of the Turing patterns for both the prey and predator populations in spatial domain $(0,200)$ are shown in Figure $8(\mathrm{~b}, \mathrm{c})$ which show that the areas with high concentration of prey population correspond to low density of predator population and vice versa.

We consider another example of patterns in the Turing-Hopf domain. Turing-Hopf domain represents the region where the homogeneous steady-state $\left(N_{*}, P_{*}\right)$ of system $(8)$ is unstable with respect to temporal and spatial perturbations. For the parameter values $a=0.3, b=0.19, c=0.2, d_{1}=2$, the Turing bifurcation threshold is found to be $d_{2}^{T}=$ 2.3039 and the corresponding $k_{T}=0.3573$. Plot of $\operatorname{Re}(\lambda)$ (red curve) confirms that the system is Turing unstable for $d_{2}=6>d_{2}^{T}$ (see Figure $9(\mathrm{a})$ ). Also at $k=0, \operatorname{Re}(\lambda)$ is positive which confirms that the parameter set lies below the Hopf bifurcation boundary in Figure 3 where $b_{H}=0.2$. Thus, the parameter set belongs to bluethe Turing-Hopf domain. The corresponding eigen mode that grows rapidly due to Turing instability has wavelength is $\frac{2 \pi}{0.31416} \approx 20$ and the approximate number of stripes to be found due to Turing instability is $\frac{1200}{20}=60$. We get 60 stripes after simulation with the given initial conditions (18) (see Figure $9(\mathrm{~b})$ ). Here also areas with high concentration of prey population correspond to low concentration of predator population and vice versa.

\subsection{Pattern diagram on $a-b$ plane}

Now we examine the effect of cross-diffusion on all types of solutions exhibited by the self-diffusion model (6). For this, we increase the cross-diffusion parameter $d_{2}$ gradually from 0 and note the changes of the regions marked in $a-b$ plane of Figure 3 . Regions I, II, III and IV are specified in the caption of Figure 3. We use the given initial conditions (18) and no-flux boundary conditions to simulate the model with cross-diffusion (8) for each parameter set of the $a-b$ plane. It is obvious that region I does not change with variation in $d_{2}$ since the co-existing steady-state remains infeasible. Conditions for Turing

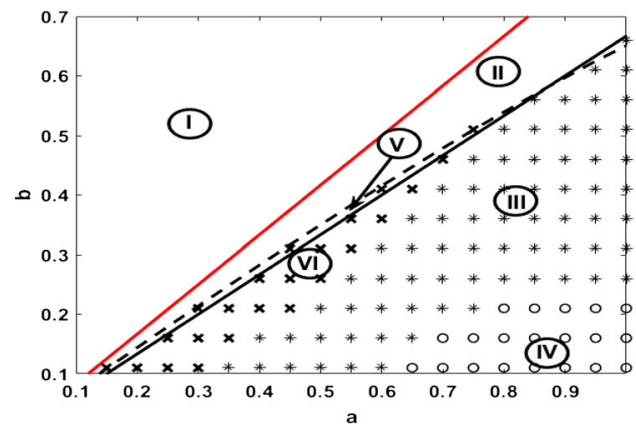

(a)

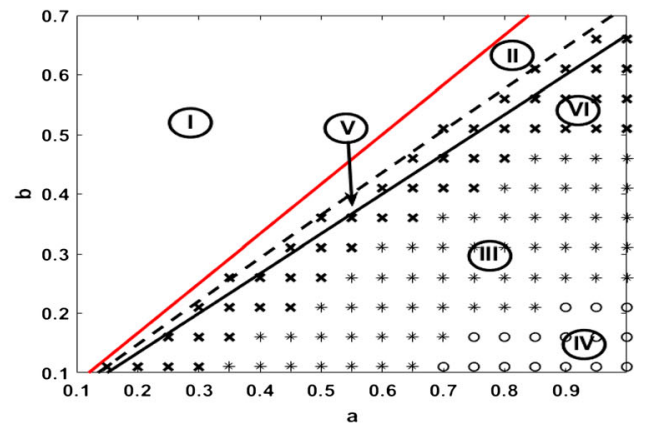

(b)

Figure 10. Bifurcation curves in $a-b$ plane for (a) $d_{2}=6$; (b) $d_{2}=12$ : red curve marks the boundary of region I; black and back dashed curves represent the Hopf and Turing bifurcation curves respectively; region II lies between the red, black and black dashed curves; ${ }^{\text {** }}$ symbols represent region III; 'o' symbols represent region IV; ' $x$ ' symbols represent stationary patterns that belong to pure Turing domain $V$ and Turing-Hopf domain VI. Other parameter values are $c=0.2, d_{1}=2$. 


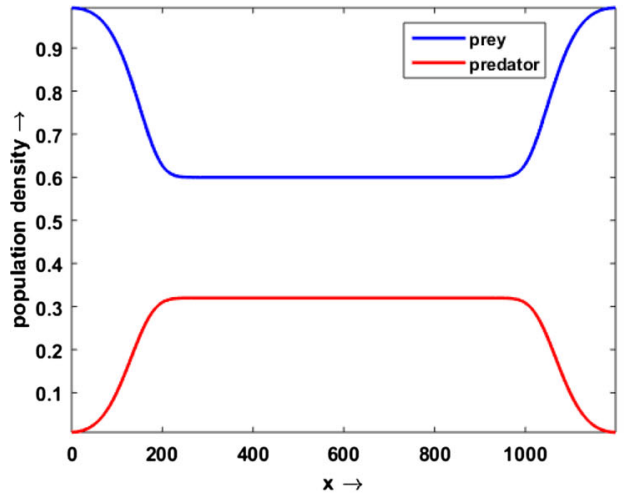

(a)

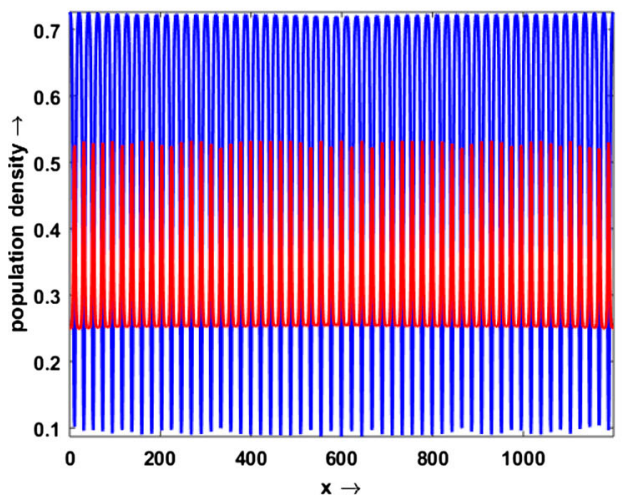

(c)

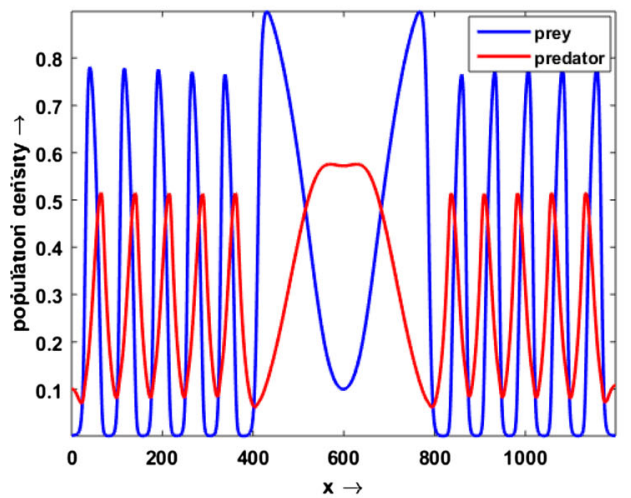

(e)

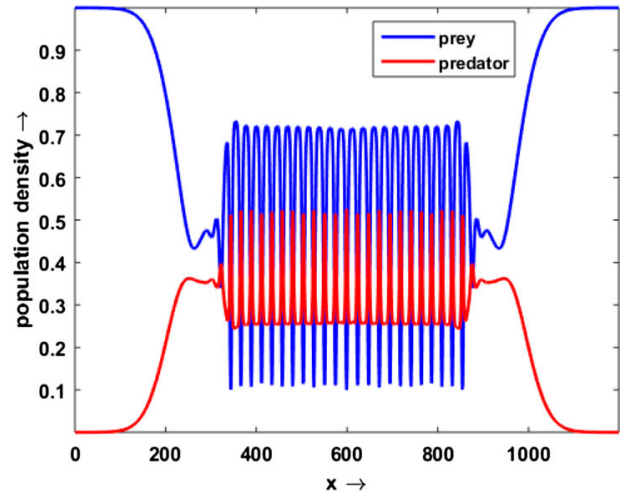

(b)

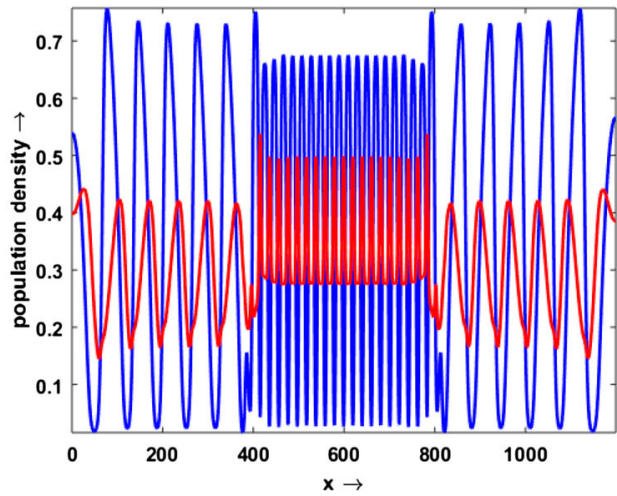

(d)

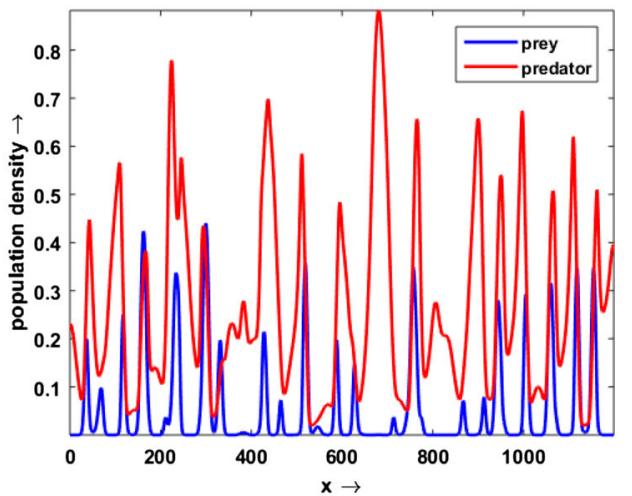

(f)

Figure 11. Plot of solutions at time $t=1500$ : (a) $a=0.2$; (b) $a=0.215$; (c) $a=0.22$; (d) $a=0.27$; (e) $a=0.35$; (f) $a=0.8$. Other parameter values are $b=0.15, c=0.2, d_{1}=2$ and $d_{2}=6$.

instability are satisfied for some parameter sets of the $a-b$ plane in presence of crossdiffusion. Hence, Turing patterns start to evolve which give rise to pure Turing domain $\mathrm{V}$ that lies between the Hopf and Turing bifurcation boundaries. We also have region VI below the Hopf bifurcation boundary that correspond to the Turing-Hopf domain in the $a-b$ plane (see Figure 10(a,b)). Regions II and III of Figure 3 become smaller in sizes due 
to addition of regions V and VI. Parameter sets giving travelling wave solutions for $d_{2}=0$ may now produce Turing patterns as $d_{2}$ increases. Also the parameter sets giving periodic in time solutions may satisfy the Turing instability conditions and produce Turing patterns as an effect of cross-diffusion. Turing patterns are produced for parameter sets around the Hopf bifurcation curve. On the other hand, chaotic patterns exhibited by parameter sets away from the Hopf bifurcation curve, become less enhanced and periodic in time solution occurs as a result of cross-diffusion. Thus, region IV also becomes smaller with an increase in $d_{2}$. The bifurcation diagrams for $d_{2}=6$ and $d_{2}=12$ in Figure $10(\mathrm{a}, \mathrm{b})$, respectively, clearly show that regions $\mathrm{V}$ and VI increase leading to diminution of regions II, III and IV with increase in $d_{2}$. It should be mentioned here that appropriate boundaries for the regions III and IV and lower boundary for region VI can not be obtained analytically rather they are identified through the numerical simulation results.

For better understanding of the change in patterns in the $a-b$ plane of Figure 10(a), we choose a particular parameter set with $b=0.15, d_{2}=6$ and increase $a$ gradually to observe the changes in the patterns. Figure 11 depicts that, travelling wave transforms into Turing patterns followed by the emergence of periodic travelling wave in the transient phase leading to periodic in time solutions with increase in $a$. Figure 11(b) represents how the travelling wave transforms into Turing patterns. Turing patterns emerge at the middle of the domain followed by travelling wave moving towards the boundary after which the whole domain is filled with Turing patterns if the simuations are run for a longer time for $a=0.215$. Turing patterns are observed at $a=0.22$ and periodic travelling waves at $a=0.35$. For some values of $a$ in between 0.22 and 0.35 , Turing like stationary patterns are observed in some parts of the domain along with periodic travelling wave in the remaining parts (see Figure 11(d)). Further increase in $a$ leads to transformation of periodic in time solutions to chaotic patterns. For Figure 10(b), we keep $a=0.8$ and $d_{2}=12$ fixed and increase $b$ gradually to observe the changes in the patterns. For this set of parameter values, chaotic patterns followed by periodic solutions, Turing patterns and travelling waves are observed.

If continuation technique for numerical simulations with respect to parameter $a$ is used keeping $b$ and $d_{2}$ fixed, various interesting phenomena are observed. In the forward continuation technique, we simulate the cross-diffusion model (8) for $a=0.181$ using initial condition (18) which gives travelling wave solution. Using the travelling wave solution as initial condition, we increase $a$ by small increment to see the changes in patterns. The process is continued using the last simulation result at each step as initial condition for the next step. Turing patterns, periodic in time solutions and chaotic solutions are observed (see Figure 12(a,b)). Periodic in time solutions are found for a larger range of $a$, than the range found when the model (8) was simulated at each value of $a$ with the given initial condition (18).

We have also used backward continuation technique for the same set of parameter values. Here, we simulate the model (8) for $a=1$ using initial conditions (18) and no-flux boundary conditions. This gives rise to chaotic solution. Next we decrease a by small decrement and use the solution for $a=1$ as initial condition. This process is continued till $a=0.181$ and the resulting patterns are noted. We observe periodic in time solutions, Turing patterns and stationary homogeneous steady state with decrease in $a$. In this case, the chaotic pattern prevails for a longer range of $a$ than it does for the case in which initial condition (18) is used for each value of $a$ (see Figure 12(c)). 


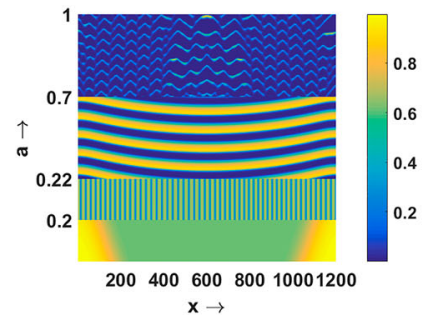

(a)

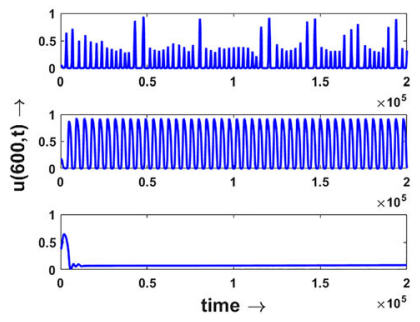

(b)

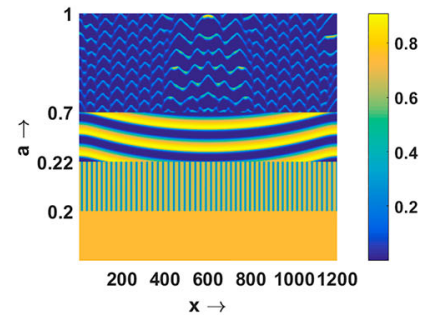

(c)

Figure 12. (a) Resulting patterns obtained for prey population by forward continuation technique for $b=0.15, d_{2}=6$ and $a \in[0.181,1]$; (b) Plot of time series for $u(600, t)$ showing chaotic pattern for $a=0.8$ (upper panel), periodic in time solutions for $a=0.4$ (middle panel) and Turing pattern for $a=0.22$ (lower panel); (c) Resulting patterns obtained for prey population by backward continuation technique for $b=0.15, d_{2}=6$ and $a \in[0.181,1]$.

\section{Conclusions}

We have considered a common prey-predator model with Rosenzweig-MacArthur type reaction kinetics as our temporal model. After deriving the Hopf bifurcation conditions for the same, we have introduced the spatio-temporal extension of the model in terms of a system of reaction-diffusion equations together with given initial conditions and no-flux boundary conditions. In addition to self-diffusion, we have considered densitydependent cross-diffusion for the predator population to include the effects of presence, absence, abundance of the prey population on the predators. Existence of Turing patterns has been investigated for the spatio-temporal model with cross-diffusion. Effects of cross-diffusion on the patterns produced by the corresponding self-diffusion model such as travelling waves, periodic travelling waves leading to periodic in time solutions, modulated periodic travelling waves leading to chaotic solutions are also elaborately studied. Main goal of this work is to explain how one type of pattern changes to another type in presence of cross-diffusion. Extensive numerical simulations are used to validate the analytical results and to explore the other possible spatio-temporal patterns like periodic and chaotic solutions.

The self-diffusion model (6) does not produce Turing patterns since the conditions for Turing instability are not satisfied. On the other hand, the Turing instability conditions can be satisfied for an appropriate parameter set in presence of cross-diffusion terms. Turing bifurcation curve which does not exist for the self-diffusion model appears in the $a-b$ parametric plane for non-zero cross-diffusion parameter $d_{2}$. Furthermore, the Turing domain and Turing-Hopf domain also increase in size with increase in the cross-diffusion parameter.

Travelling wave solutions are produced by the self-diffusion model (6) connecting two or three homogeneous steady-states. The solutions may remain same or may bifurcate to Turing patterns due to inclusion of cross-diffusion terms. In case of similar solution, the speed of the travelling wave increases with an increase in the cross-diffusion parameter. Also the Turing patterns depict that areas with high concentration of prey population correspond to low predator concentration and vice versa which is ecologically realistic since the prey population tend to be away from regions where high concentration of predator 
population is present. Also the predators gather near the boundary of the regions where prey population is high to catch hold of the same.

For some sets of parameter values just below the Hopf bifurcation curve in the $a-b$ parametric plane, the model with self-diffusion (6) generates periodic in time solutions which are converted into Turing patterns as well as into a combination of Turing patterns and periodic travelling waves as the cross-diffusion parameter is introduced and gradually increased. For the parameter values below and away from the Hopf bifurcation curve, chaotic solutions are produced by the self-diffusion model (6). Numerical simulations for some of these parameter sets lead to conversion of chaotic patterns into periodic in time solutions. Periodic in time solutions prevail over a larger range of parameters when forward continuation technique is used in simulations whereas the same occurs for chaotic patterns in the backward continuation procedure.

Travelling waves and periodic in time solutions produced by the self-diffusion model (6) convert into Turing patterns as a result of cross-diffusion even for the case where the self-diffusion parameters are same for both the prey and predator populations. For lower values of self-diffusion parameter, the speed of the travelling wave increases first with increase in cross-diffusion parameter before it attains a saturation level. For higher values of self-diffusion parameter, the speed of the travelling wave is almost independent of the cross-diffusion parameter. Chaotic patterns generated by the self-diffusion model (6) may get subdued and periodic in time solutions appear as a result of cross-diffusion. The temporal model shows global stability for predator free equilibrium point as well as stable limit cycle under proper parametric restriction and the corresponding spatio-temporal model with self-diffusion shows patterns which are variable with respect to time. However, it is possible to find solutions which are stationary with respect to time due to introduction of cross-diffusion terms. Thus, density dependent cross-diffusion has significant effects on the pattern formation scenario of the model with self-diffusion and is solely responsible for the formation of Turing patterns in prey-predator model with Holling type II functional response.

Thus, travelling wave, periodic in time solutions and chaotic patterns are observed under certain parametric conditions in the self-diffusion model. On the other hand, Turing pattern along with the combination of Turing pattern and periodic travelling wave, are additional patterns that are observed in presence of cross-diffusion. This approach can be extended to two spatial dimensions for other prey-predator models with different functional responses and a comparative study of the pattern formation scenario can be explored. Also, other forms of cross-diffusion terms can be used and the resulting patterns can be studied as well.

\section{Disclosure statement}

No potential conflict of interest was reported by the authors.

\section{References}

Aragón, J. L., Barrio, R. A., Woolley, T. E., Baker, R. E., \& Maini, P. K. (2012). Nonlinear effects on turing patterns: Time oscillations and chaos. Physical Review E, 86(2), 026201.

Cross, M. C., \& Hohenberg, P. C. (1993). Pattern formation outside of equilibrium. Reviews of Modern Physics, 65(3), 851. 
Dunbar, S. (1983, May). Travelling wave solutions of diffusive lotka-volterra equations. Journal of Mathematical Biology, 17(1), 11-32.

Dunbar, S. (1984). Traveling wave solutions of diffusive lotka-volterra equations: A heteroclinic connection in $\mathbb{R}^{4}$. Transactions of the American Mathematical Society, 286(2), 557-594.

Dunbar, S. (1986). Traveling waves in diffusive predator-prey equations: Periodic orbits and pointto-periodic heteroclinic orbits. SIAM Journal on Applied Mathematics, 46(6), 1057-1078.

Feltham, D. L., \& Chaplain, M. A. J. (2000). Travelling waves in a model of species migration. Applied Mathematics Letters, 13(7), 67-73.

Gambino, G., Lombardo, M. C., \& Sammartino, M. (2013). Pattern formation driven by crossdiffusion in a 2d domain. Nonlinear Analysis: Real World Applications, 14(3), 1755-1779.

Gambino, G., Lombardo, M. C., \& Sammartino, M. (2018, January). Cross-diffusion-induced subharmonic spatial resonances in a predator-prey system. Physical Review E, 97, 012220.

Gardner, R. (1984). Existence of travelling wave solutions of predator-prey systems via the connection index. SIAM Journal on Applied Mathematics, 44(1), 56-79.

Garvie, M. R., \& Trenchea, C. (2010). Spatiotemporal dynamics of two generic predator-prey models. Journal of Biological Dynamics, 4(6), 559-570. PMID: 22881204.

Hilker, F. M., \& Lewis, M. A. (2010, August). Predator-prey systems in streams and rivers. Theoretical Ecology, 3(3), 175-193.

Holling, C. S. (1965). The functional response of predators to prey density and its role in mimicry and population regulation. Memoirs of the Entomological Society of Canada, 97(S45), $5-60$.

Hong, K., \& Weng, P. (2013). Stability and traveling waves of a stage-structured predator-prey model with holling type-ii functional response and harvesting. Nonlinear Analysis: Real World Applications, 14(1), 83-103.

Huang, J., Lu, G., \& Ruan, S. (2003, February). Existence of traveling wave solutions in a diffusive predator-prey model. Journal of Mathematical Biology, 46(2), 132-152.

Huang, W. (2016). A geometric approach in the study of traveling waves for some classes of nonmonotone reaction-diffusion systems. Journal of Differential Equations, 260(3), 2190-2224.

Huang, Y., \& Weng, P. (2013). Traveling waves for a diffusive predator-prey system with general functional response. Nonlinear Analysis: Real World Applications, 14(2), 940-959.

Huang, Y.-L., \& Lin, G. (2014). Traveling wave solutions in a diffusive system with two preys and one predator. Journal of Mathematical Analysis and Applications, 418(1), 163-184.

Iida, M., Mimura, M., \& Ninomiya, H. (2006). Diffusion, cross-diffusion and competitive interaction. Journal of mathematical biology, 53(4), 617-641.

Lee, J. M., Hillen, T., \& Lewis, M. A. (2008, April). Continuous traveling waves for prey-taxis. Bulletin of Mathematical Biology, 70(3), 654-676.

Li, W.-T., \& Wu, S.-L. (2008). Traveling waves in a diffusive predator-prey model with holling type-iii functional response. Chaos, Solitons \& Fractals, 37(2), 476-486.

Lin, G. (2014). Invasion traveling wave solutions of a predator-prey system. Nonlinear Analysis: Theory, Methods \& Applications, 96, 47-58.

Lin, X., Weng, P., \& Wu, C. (2011, December). Traveling wave solutions for a predator-prey system with sigmoidal response function. Journal of Dynamics and Differential Equations, 23(4), 903-921.

Ling, Z., Zhang, L., \& Lin, Z. (2014). Turing pattern formation in a predator-prey system with cross diffusion. Applied Mathematical Modelling, 38(21), 5022-5032.

Liu, J., \& Lin, Z. (2010). Stationary patterns for a predator-prey model with holling type iii response function and cross-diffusion. Bulletin of the Korean Mathematical Society, 47(2), 251-261.

Mimura, M., \& Kawasaki, K. (1980). Spatial segregation in competitive interaction-diffusion equations. Journal of Mathematical Biology, 9(1), 49-64.

Morales, J. M., Moorcroft, P. R., Matthiopoulos, J., Frair, J. L., Kie, J. G., Powell, R. A., Merrill, E. H., \& Haydon, D. T. (2010). Building the bridge between animal movement and population dynamics. Philosophical Transactions of the Royal Society of London B: Biological Sciences, 365(1550), 2289-2301. 
Murray, J. D. (2001). Mathematical biology. II spatial models and biomedical applications \{Interdisciplinary Applied Mathematics V. 18\}. New York: Springer-Verlag New York Incorporated.

Nathan, R., Getz, W. M., Revilla, E., Holyoak, M., Kadmon, R., Saltz, D., \& P. E. Smouse (2008). A movement ecology paradigm for unifying organismal movement research. In Proceedings of the national academy of sciences.

Oeda, K. (2011). Effect of cross-diffusion on the stationary problem of a prey-predator model with a protection zone. Journal of Differential Equations, 250(10), 3988-4009.

Okubo, A., \& Levin, S. A. (2013). Diffusion and ecological problems: Modern perspectives (Vol. 14). New York: Springer Science \& Business Media.

Pascual, M. (1993). Diffusion-induced chaos in a spatial predator-prey system. Proceedings of the Royal Society of London B: Biological Sciences, 251(1330), 1-7.

Peng, R., Shangbing, A., \& Yihong, D. (2017). Traveling waves for a generalized holling-tanner predator-prey model. Journal of Differential Equations, 263(11), 7782-7814.

Peng, Y., \& Zhang, T. (2016). Turing instability and pattern induced by cross-diffusion in a predatorprey system with allee effect. Applied Mathematics and Computation, 275, 1-12.

Perko, L. (2013). Differential equations and dynamical systems (Vol. 7). New York: Springer Science \& Business Media.

Petrovskii, S. V., \& Malchow, H. (1999). A minimal model of pattern formation in a prey-predator system. Mathematical and Computer Modelling, 29(8), 49-63.

Potts, J. R., \& Petrovskii, S. V. (2017). Fortune favours the brave: Movement responses shape demographic dynamics in strongly competing populations. Journal of Theoretical Biology, 420, 190-199.

Rosenzweig, M. L., \& MacArthur, R. H. (1963). Graphical representation and stability conditions of predator-prey interactions. The American Naturalist, 97(895), 209-223.

Ruiz-Baier, R., \& Tian, C. (2013). Mathematical analysis and numerical simulation of pattern formation under cross-diffusion. Nonlinear Analysis: Real World Applications, 14(1), 601-612.

Sherratt, J. A. (1998). Invading wave fronts and their oscillatory wakes are linked by a modulated travelling phase resetting wave. Physica D: Nonlinear Phenomena, 117(1), 145-166.

Sherratt, J. A., Eagan, B. T., \& Lewis, M. A. (1997). Oscillations and chaos behind predator-prey invasion: Mathematical artifact or ecological reality? Philosophical Transactions of the Royal Society of London B: Biological Sciences, 352(1349), 21-38.

Sherratt, J. A., Lambin, X., \& Sherratt, T. N. (2003). The effects of the size and shape of landscape features on the formation of traveling waves in cyclic populations. The American Naturalist, 162(4), 503-513.

Shigesada, N., Kawasaki, K., \& Teramoto, E. (1979). Spatial segregation of interacting species. Journal of Theoretical Biology, 79(1), 83-99.

Shigesada, N., Kawasaki, K., \& Teramoto, E. (1986). Traveling periodic waves in heterogeneous environments. Theoretical Population Biology, 30(1), 143-160.

Turing, A. M. (1952). The chemical basis of morphogenesis. Philosophical Transactions of the Royal Society of London. Series B, Biological Sciences, 237(641), 37-72.

Volpert, V., \& Petrovskii, S. (2009). Reaction-diffusion waves in biology. Physics of Life Reviews, 6(4), 267-310.

Zhang, T., Wang, W., \& Wang, K. (2016). Minimal wave speed for a class of non-cooperative diffusion-reaction system. Journal of Differential Equations, 260(3), 2763-2791. 